\title{
So antikoagulieren Sie maßgeschneidert
}

\author{
Patienten mit erhöhtem Thromboserisiko brauchen besondere ärztliche Aufmerksamkeit und bei \\ Bedarf die richtige Antikoagulation. Was bei älteren, bei krebskranken und bei adipösen Patienten \\ zu beachten ist, erläutert Dr. Peter Stiefelhagen in dieser Gerinnungssprechstunde.
}

? Ich betreue eine 76-jährige multimorbide, immobile Patientin, bei der vor einem halben Jahr eine spontane tiefe Beinvenenthrombose aufgetreten war. Die daraufhin eingeleitete Antikoagulation mit Marcumar ${ }^{\circledR}$ musste zweimal kurz wegen Zahnproblemen und einer Kataraktoperation unterbrochen werden. Blutungskomplikationen traten nicht auf. Die Patientin gibt an, dass bei ihr vor ca. 35 Jahren schon einmal eine "leichte oberflächliche Thrombose" aufgetreten sei. Soll ich die Antikoagulation nun beenden oder weiterführen? Und wenn ja, womit? Marcumar weiter oder NOAK oder reicht ASS?

Aus der Anamnese lässt sich nicht sicher beurteilen, ob damals wirklich eine tiefe Beinvenenthrombose vorlag oder nur

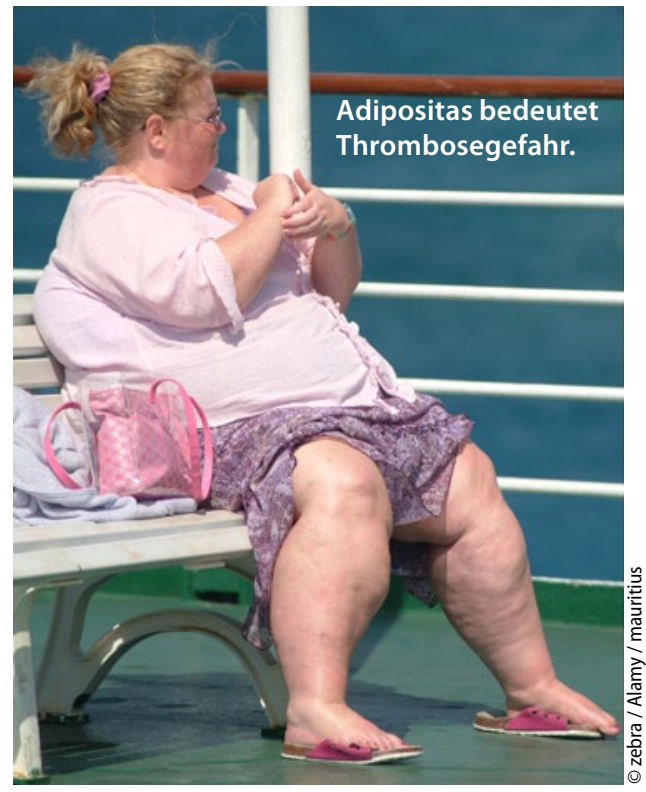

eine oberflächliche Thrombophlebitis. Insgesamt ist aber von einem erhöhten Risiko für eine Rezidivthrombose auszugehen, sodass man eine Fortführung der Antikoagulation diskutieren sollte, zumal darunter keine Blutungskomplikationen aufgetreten sind. Allerdings ist auch zu bedenken, dass das Risiko einer Blutung mit dem Alter deutlich steigt.

Sie haben nun mehrere Möglichkeiten. Wenn Sie weiter den Vitamin-K-Antagonisten geben möchten, sollte der Ziel-INR-Wert bei 2-2,5 liegen. Ein Zielwert von 1,5-2 ist unzureichend. Wenn Sie auf ASS umstellen, wird das Risiko für eine Rezidivthrombose von 11,6\% (unter Placebo) auf 6,6\% gesenkt. Die Blutungsrate liegt im Placebobereich. Die Wirkung ist aber deutlich schwächer als bei Gabe eines Antikoagulans. Für die NOAK, genauer gesagt für Apixaban, gibt es erste Daten, dass nach sechs Monaten eine Dosisreduktion von 2 x $5 \mathrm{mg}$ auf 2 × 2,5 mg die Wirkung im Hinblick auf das Rezidivrisiko nicht schmälert, aber das Blutungsrisiko deutlich vermindert; es liegt dann im Placebobereich.

$?$

Wie muss die medikamentöse Thromboseprophylaxe bei stark übergewichtigen Patienten aussehen? Reicht die Standarddosierung?

Adipöse Patienten haben per se ein mindestens zweifach erhöhtes Thromboserisiko. Bei einem operativen Eingriff oder einer immobilisierenden internistischen Erkrankung steigt das Risiko nochmals deutlich an. Für die niedermolekularen Heparine $(\mathrm{NMH})$ gibt es bisher keine klaren Dosisempfehlungen für den Einsatz bei morbid Adipösen. Es empfiehlt sich eine Dosiserhöhung, wobei allerdings ein Monitoring des Anti-Xa-Spiegels erfolgen sollte. Für die NOAK haben kleine pharmakologische Studien gezeigt, dass die maximalen Plasmaspiegel gleich bzw. nur gering unterschiedlich sind, d.h. auch bei Adipösen scheint eine ausreichende Wirkung gesichert, sodass keine Dosisanpassung empfohlen wird.

\section{Es heißt immer: Tumorpatienten haben ein stark erhöhtes Throm-} boserisiko und sollten deshalb eine Prophylaxe vorzugsweise mit NMH erhalten. Muss ich bei allen meinen Tumorpatienten eine lebenslange Thromboseprophylaxe durchführen?

Diese Empfehlung gilt vorrangig für stationäre Patienten, v. a. dann, wenn eine Intervention bzw. Operation erfolgt. Bei ambulanten Patienten wird keine routinemäßige Prophylaxe empfohlen. Sie ist jedoch sinnvoll und notwendig, wenn passagere Risiken, wie Bettlägerigkeit bei einem Infekt, dazukommen.

\section{?}

Gibt es Kontraindikationen für eine medikamentöse Thromboseprophylaxe?

Ja, die gibt es! Dazu zählen Heparin-induzierte Thrombozytopenie (HIT Typ 2), stark erhöhte Blutungsneigung etwa bei Leber- oder Niereninsuffizienz, schwere Thrombozytopenie, aktive Blutung, Endokarditis und drohende Fehlgeburt.

Dr. med. Peter Stiefelhagen 